\title{
An investigation on effect of EDL on heat transfer of micro heat pipe with square and triangular cross section
}

\author{
Maryam Fallah Abbasi ${ }^{1}$, Hossein Shokouhmand ${ }^{2, *}$, and Morteza Khayat ${ }^{1}$ \\ ${ }^{1}$ Department of Mechanical Engineering, Science and Research Branch, Islamic Azad University, Tehran, Iran \\ ${ }^{2}$ School of Mechanical Engineering, College of Eng. University of Tehran, Tehran, Iran
}

Received: 8 December 2017 / Accepted: 9 February 2020

\begin{abstract}
Electronic industries have always been trying to improve the efficiency of electronic devices with small dimensions through thermal management of this equipment, thus increasing the use of small thermal sinks. In this study micro heat pipes with triangular and square cross sections have been manufactured and tested. One of the main objectives is to obtain an understanding of micro heat pipes and their role in energy transmission with electrical double layer (EDL). Micro heat pipes are highly efficient heat transfer devices, which use the continuous evaporation/condensation of a suitable working fluid for two-phase heat transport in a closed system. Since the latent heat of vaporization is very large, heat pipes transport heat at small temperature difference, with high rates. Because of variety of advantage features these devices have found a number of applications both in space and terrestrial technologies. The theory of operation micro heat pipes with EDL is described and the micro heat pipe has been studied. The temperature distribution have achieved through five thermocouples installed on the body. Water and different solution mixture of water and ethanol have used to investigate effect of the electric double layer heat transfer. It was noticed that the electric double layer of ionized fluid has caused reduction of heat transfer.
\end{abstract}

Keywords: heat transfer / micro heat pipe / electrical double layer / condensation / evaporation

\section{Introduction}

Components, mechanical and electrical devices with micron-size are becoming more common in commercial applications and scientific research. Rapid progress in micro-electric has created a basic revolution in computers, electronics devices, communications systems and many other tools. Lightweight, compact and high-performance of micro-systems will have important applications in transportation, the environment and space research, biochemical and other industries of chemical process. The main advantages of these systems and micro-fluids are including high rates of heat transfer, mass transfer and chemical reactions and reduced size of the samples [1].

With increasing heat dissipation of micro-electronic devices and reduced size of them, thermal management becomes a more important element than the electronic product design. So, long life and reliable performance of parts may be achieved by effective control of device operating temperature with ranges adjustment by

\footnotetext{
* e-mail: ho.shokouhmand@gmail.com
}

engineering design. Heat sinks are devices that facilitate the release of heat from hot surfaces that usually, transfer the heat generated by the components to the cooler environment. The primary objective of the heat sink is keeping the device temperature in the lower the maximum temperature allowed by the manufacturer.

Mainly, micro devices have a different behavior than macro devices. That it can be attributed to several factors, among this is electro-kinetic effects. Electro-kinetic transport applications in micro and nano-dimension are important. Electric double layer (EDL) in the containing ion flows has significant effects on Heat transfer and fluid flow inside the micro heat pipes. So that the usual equations of heat transfer and flow in the channels about micro heat pipe loses its credibility [2].

Induced surface charge at the interface between solid and liquid phase causes the formation of an electrical double layer and the non-uniform distribution of fluid ions on the surface. In transfer of an electrolyte by pressure-driving way inside a micro-pipe, electrical double layer formation and movement of loads in it along with applied fluid creates electric current which is called streaming. 
In the past decades, a number of cooling system designs including simple flat thermal loser and more complex, such as two-phase heat sink cooling micro-jet have been proposed and widely have been studied experimentally and theoretically. Knight et al. [3] worked in the field of allowed optimization micro-channel heat sink by reducing the aspect ratio, the ratio of the width of the blade channel, Nusselt number and type of flow and volumetric rate of flow. Groll et al. [4] have investigated number of articles about the micro heat pipes for thermal control of electronic equipment. They have compared and made microclassification more than 100 articles about sinks heat pipe based on their performance, their design, and their thermal physical parameters. Applied fluids and wall material is discussed and combination possibilities are offered. Le Berre et al. [5] accomplished experimental study on the performance of an array of micro heat pipe for different charge of liquid in different experimental conditions. Micro heat pipe dimensions are $20 \mathrm{~mm} \times 20 \mathrm{~mm}$ and contain 27 parallel channels with a triangular cross section channel, width and depth of 350-500, respectively.

\section{Material and method}

\subsection{Governing equation}

Micro heat pipes function is much like conventional heat pipes. Vaporizing of working fluid in the evaporator result in heat absorption from the heat source and Steam move toward condenser and in there condense and release its latent heat. Micro heat pipes, contrary to conventional heat pipes, use grooves or sharp edges to return the fluid from the evaporator to the condenser, Instead of using porous media (Fig. 1).

Solution area has two phases of Vapor and liquid. In addition, electrical area which is existed has influence on solution area. To track the interface between the liquid and vapor phase Lagrangian and Eulerian methods is available. Lagrangian method scope is limited to flows in which changing in surface form are not extreme. Lagrangian method is less accurate than Eulerian methods. But it can use to simulate flows with complex interface (here vapourliquid). One of the most famous Eulerian method is VOF or volume of fluid method that defined by volume fraction of fluid in the cell network computing solutions and it's expressed by $\alpha$. If the cell not containing fluid, the amount of $\alpha$ is zero. And if it is completely fluid filled, the amount of $\alpha$ is one. And if part of it is filled, $\alpha$ is between zero and one. In this way, the contact area between the liquid and vapor phases will be achieved as shown in Figure $2[2,6,7]$.

\subsection{Navier-Stokes equations}

The conservation equations in the single-field representation must account for differences in material properties of individual fluids as well as surface tension acting at the interface. For the entire flow field, the continuity and momentum equations are expressed as:

$$
\frac{\partial \rho}{\partial t}+\nabla \cdot(\rho v)=0
$$

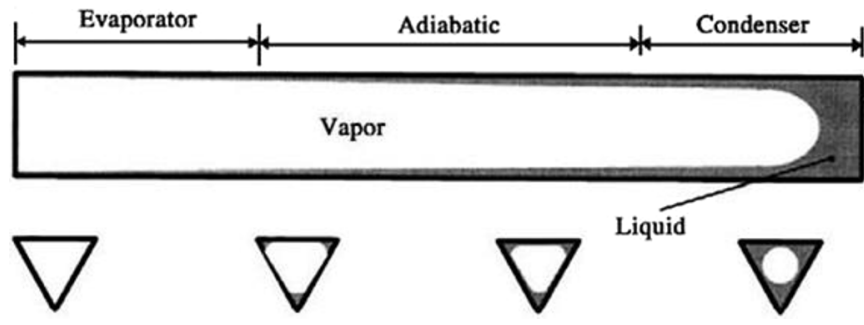

Fig. 1. Schematic of the micro heat pipe function and utilizing groove to return the fluid from the evaporator to the condenser [16].

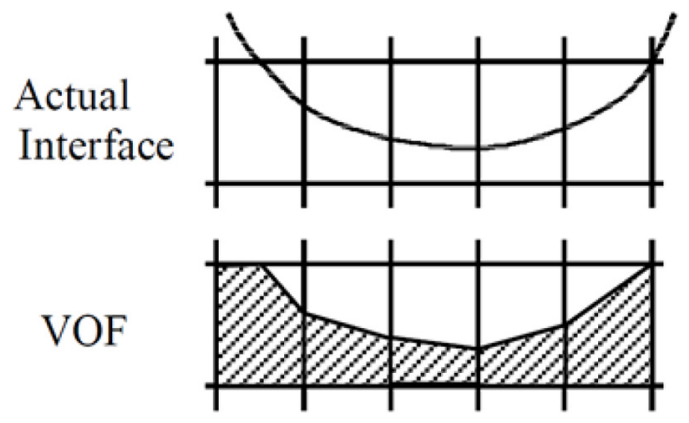

Fig. 2. Contact area of two phases in real mode (above) and by Eulerian VOF method (bottom) [17].

$$
\frac{\partial}{\partial t}(\rho v)+\nabla \cdot(\rho v v)=-\nabla p+\nabla \cdot \tau+\rho g+F+\underbrace{\sigma \kappa \nabla \alpha}_{\text {effect of capillary atraction }}
$$

where $v$ is velocity, $\tau$ is the rate of strain tensor, $p$ is pressure. The last term in equation (2) represents the source of momentum due to surface tension effects.

The stress tensor $\tau$ is expressed as:

$$
\tau=\mu\left(\nabla v+\nabla v^{T}\right)
$$

where $\mu$ is the dynamic viscosity of the "mixture" and $\nabla v^{T}$ is the transpose of the

$$
\begin{aligned}
\nabla \cdot \tau & =\nabla \cdot\left[\mu\left(\nabla v+\nabla v^{T}\right)\right]=\nabla \cdot(\mu \nabla v)+\nabla \cdot\left(\mu \nabla v^{T}\right) \\
& =\nabla \cdot(\mu \nabla v)+(\nabla v) \cdot(\nabla \mu)+\mu \nabla(\nabla \cdot v) \\
& =\nabla \cdot(\mu \nabla v)+(\nabla v) \cdot \nabla \mu
\end{aligned}
$$

$$
\begin{aligned}
\frac{\partial}{\partial t}(\rho v)+\nabla \cdot(\rho v v)= & -\nabla p+\nabla \cdot(\mu \nabla v)+(\nabla v) \cdot \nabla \mu+\rho g \\
& +F+\sigma \kappa \nabla \vec{n}
\end{aligned}
$$

where $T$ is vector transpose of matrix and $\mu$ is dynamic viscosity of the mixture and at the end the momentum equation which is a suitable equation for micro pipes would be [8]. 


\subsection{VOF equation}

VOF equation based on transmission of the volume fraction $\alpha$ is written as follows:

$$
\frac{\partial \alpha}{\partial t}+\nabla \cdot(v \alpha)=0
$$

and physical properties of the mixture would be as follows:

$$
\begin{gathered}
\rho=\alpha \rho_{l}+(1-\alpha) \rho_{g} \\
\mu=\alpha \mu_{l}+(1-\alpha) \mu_{g} .
\end{gathered}
$$

However, according to both liquid and vapor phase, Eulerian equation VOF method is based on the volume fraction of $\alpha$ and the velocity of liquid and vapor phases are as follows $[6,9,10]$ :

$$
\begin{gathered}
\frac{\partial \alpha}{\partial t}+\nabla \cdot\left(v_{l} \alpha\right)=0 \\
\frac{\partial(1-\alpha)}{\partial t}+\nabla \cdot\left[v_{g}(1-\alpha)\right]=0 .
\end{gathered}
$$

The indexes $l$ and $g$ respectively represent the phase velocity of the liquid and vapor areas. According to the Expression, a weight expression for total velocity based on velocity of the liquid and vapor phases can be provided as follows:

$$
v=\alpha v_{l}+(1-\alpha) v_{g} .
$$

The general VOF equation for the two-phase vaporliquid area is as follows:

$$
\frac{\partial \alpha}{\partial t}+\nabla \cdot(v \alpha)+\nabla \cdot\left[v_{r} \alpha(1-\alpha)\right]=0
$$

\subsection{Energy equation}

$$
\frac{\partial}{\partial t}\left(\rho c_{p} T\right)+\nabla \cdot\left(v\left(\rho c_{p} T+p\right)\right)+\nabla \cdot\left(k_{e f f} \nabla T\right)+S_{h} .
$$

In the above equation, the term $S_{h}$ is any source of heat such as radiation and $k_{\text {eff }}$ is effective thermal conductivity which it were calculated for the cell contains two phases as equations (4) and (5), and $T$ is the temperature and is calculated for the liquid and vapor phases using the following formula:

$$
T=\alpha T_{l}+(1-\alpha) T_{g} .
$$

\subsection{Conductive heat transfer (wall)}

The wall has conductive heat transfer and its governing equation is [6]:

$$
q^{\prime \prime \prime}+\nabla\left(k_{s} \nabla T\right)=\left(\partial C_{p}\right)_{s} \frac{\partial T}{\partial t},
$$

where $q^{\prime \prime \prime}$ is the heat generated by the heat source and $k_{\mathrm{s}}$ is wall heat transfer coefficient (solid) and $C_{p}$ is specific heat.

\subsection{The effect of body force}

If $\phi$ be the external electric potential on micro heat pipe, the body force which is caused by that is written as follows $[6,11]$ :

$$
\begin{gathered}
f_{1}=\rho_{e} E=\rho_{e} \nabla \phi \\
\rho_{e}=\varepsilon \varepsilon_{0} k^{2} \psi_{1} \\
k=\sqrt{\frac{F^{2}}{\varepsilon R T} \sum_{i} z_{i}^{2} c_{i, 0} .}
\end{gathered}
$$

In the above equations, $k$ is inverse of Debye length and $F$ is Faraday constant and $R$ is the gases constant and $T$ is the absolute temperature and $c_{i, 0}$ the mass concentration of ion $i$ and $z_{i}$ is $i$-Valence of particle and $\psi_{1}$ is potential of the wall flux. Electric potential $\Psi_{2}$ and volumetric charge density $\rho_{e}$ of the electric double layer, caused by liquid and solid contacts with the condition of symmetry, are expressed by the Poisson-Boltzmann equation as follows $[12,13]$.

$$
\begin{gathered}
\nabla^{2} \psi_{2}=-\frac{2 n_{0} z e}{\varepsilon} \sinh \left(-\frac{z e \psi_{2}}{k_{b} T}\right) \\
\rho_{e}=-\varepsilon \nabla^{2} \psi_{2}=-2 n_{0} z e \sinh \left(-\frac{z e \psi_{2}}{k_{b} T}\right)
\end{gathered}
$$

where $\varepsilon$ is the dielectric constant and $n_{0}$ is bulk ionic concentration and $z$ is ions valence and $e$ is protons flux and $k_{b}$ is Boltzmann constant and $T$ is absolute temperature. Body force, which is caused by electric double layer between the liquid and solid phases, is obtained from the following equation

$$
f_{2}=\rho_{e} E
$$

and $E$ is the electro-viscous force. Body force, which is caused by electric double layer between the liquid and vapor phases, is as follows [14]:

$$
\begin{aligned}
& f_{1}=\underbrace{-\frac{a}{2} \psi_{3}^{2}+\frac{b}{4} \psi_{3}^{4}-c z_{i} e V_{e} \psi_{3}}_{\text {Homogeneous system }}+\underbrace{\frac{d}{2}\left(\nabla \psi_{3}\right)^{2}}_{\text {Non-homogeneous system }} \\
& a=\frac{U_{\text {sum }}-4 k_{s} T}{l^{3}}, \quad b=\frac{16 k_{s} T}{3 l^{3}}, \quad c=\frac{1}{2 l^{3}}, \quad d=\frac{U_{\text {sum }}}{2 l^{3}}
\end{aligned}
$$

where $U_{\text {sum }}$ is concerned to intermolecular potential and $z_{i}$ is the charge number and $e$ is initial charge and $V_{e}$ is the electrostatic potential and value of $\psi_{3}$ achieved by 
following formula [20]

$$
\mu^{S}=\mu^{-S}+q \psi_{3} .
$$

In the above equation, $\mu^{S}$ is the chemical potential of ions at the interface and $q$ is the value of ion charge. In the present research, apart from the gravity, there are electric forces that affect the momentum. That all these forces are:

$$
F=f_{1}+f_{2}+f_{3} .
$$

\section{Set-up used in research}

Making of micro heat pipe was significantly complex because of its small dimension. In this research, the micro copper pipe with triangular and square cross section were used which were prepared from commercially cylindrical pipe which was available. Due to the need to determine the exact temperature in the desired areas, cross section and the pipe diameter before deformation was brought to the desired dimensions by using heat and tension (Figs. 3 and 4; Tab. 1).

After the design and manufacturing of micro pipes, quality of working fluid charge is very important. Presence of non-condensable gases leads to disorder of fluid flow inside the heat pipe and disrupt the performance of heat pipes. At worst condition completely stopped the flow of fluid and the performance of heat pipe and heat pipe acts as a conductor.

To avoid this situation, in manufacturing process of required samples, firstly the end of micro heat pipe with a length longer than samples in above table was completely blocked and suction was fulfilled from other side. Then working fluid is injected as amount as $20 \%$ of volume of required micro pipe and leakage test was conducted after severing additional section and blocking the beginning of micro pipe.

Test equipment for thermal performance of MHP consists of a vacuum chamber and a heat transfer system to cool the condenser. Evaporator consists of a heater with a 0.36 diameter wire as heat source. This heater is settled around the body of copper pipe for providing a constant thermal load and is connected and linked to the evaporator wall via an external link. The condenser is cooled by the circulation of water flow. Vacuum chamber which is made of acrylic material to minimize heat loss to the environment was used that is shown in Figure 5.

$\mathrm{K}$ type Thermocouples $(0.08 \mathrm{~mm})$ are located by soldering in two section on the evaporator wall, one point in the adiabatic section, and two points in the condenser. The locations of the thermocouples are shown in Schematic of test set up as X that these dimensions are for the MHP with a $50 \mathrm{~mm}$ total length (Figs. 5 and 6 ). An adapter has been used to set the sensors (Fig. 7).

MHP is made of oxygen-free copper and its wall thickness is between 0.27 and $0.28 \mathrm{~mm}$ pure water and also ordinary water and different solution mixture of water and

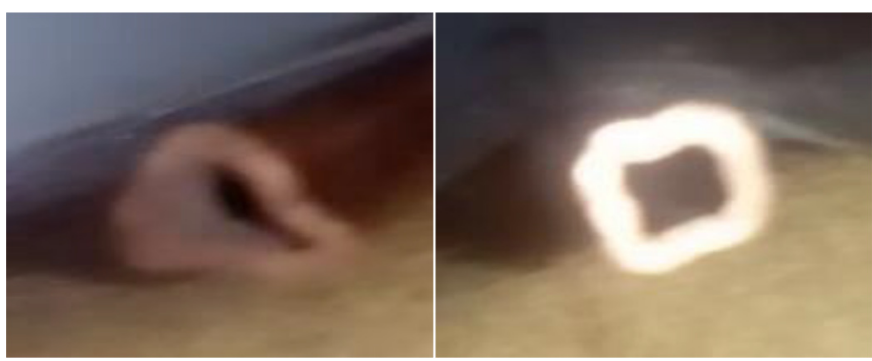

Fig. 3. Cross section of micro heat pipe (triangular and square curves type).

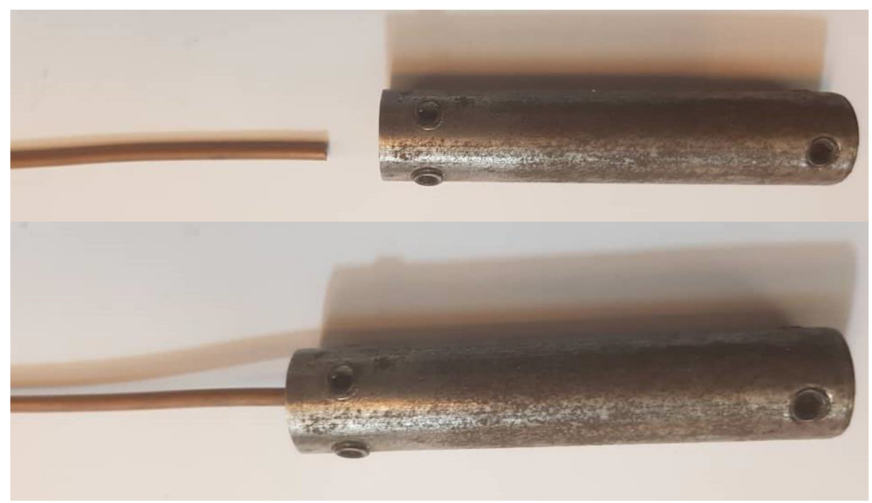

Fig. 4. Special tool designed to form a pipe to the triangular and square cross-section.

Table 1. Characteristics of examples of micro heat pipes in experimental test.

\begin{tabular}{ll}
\hline Triangular \& square & Shape of cross section \\
\hline 50 & Total length of sample \\
$10(\mathrm{~mm})$ & Length of evaporator \\
$15(\mathrm{~mm})$ & Length of adiabatic section \\
$25(\mathrm{~mm})$ & Length of condenser \\
Working fluid & $\begin{array}{l}\text { Water/Different solution mixture } \\
\text { of water and ethanol }\end{array}$ \\
Quality of micro pipe & Oxygen-free copper \\
\hline
\end{tabular}

ethanol as a working fluid is used, which has a relatively large surface tension and Specific latent heat between 30 and $160{ }^{\circ} \mathrm{C}$ operating temperature. Filling ratio of fluid is $20 \%$ of the internal volume of the MHP in which steam is not possible, may occur in the condenser. Heat transfer by changing working fluid phase could not be completed in blocked fluid region. The temperature of end of the condenser is $5-20^{\circ} \mathrm{C}$ less than the area adjacent to the condenser, and heat transfer rate of MHP due to blocking of the liquid has dropped. Thus, inactive amount of working fluid due to the closure of the design phase MHP should be considered for the calculation of filling ratio of fluid. The details of blocking mechanism have been not reported in any paper and still remain an underdeveloped subject. 

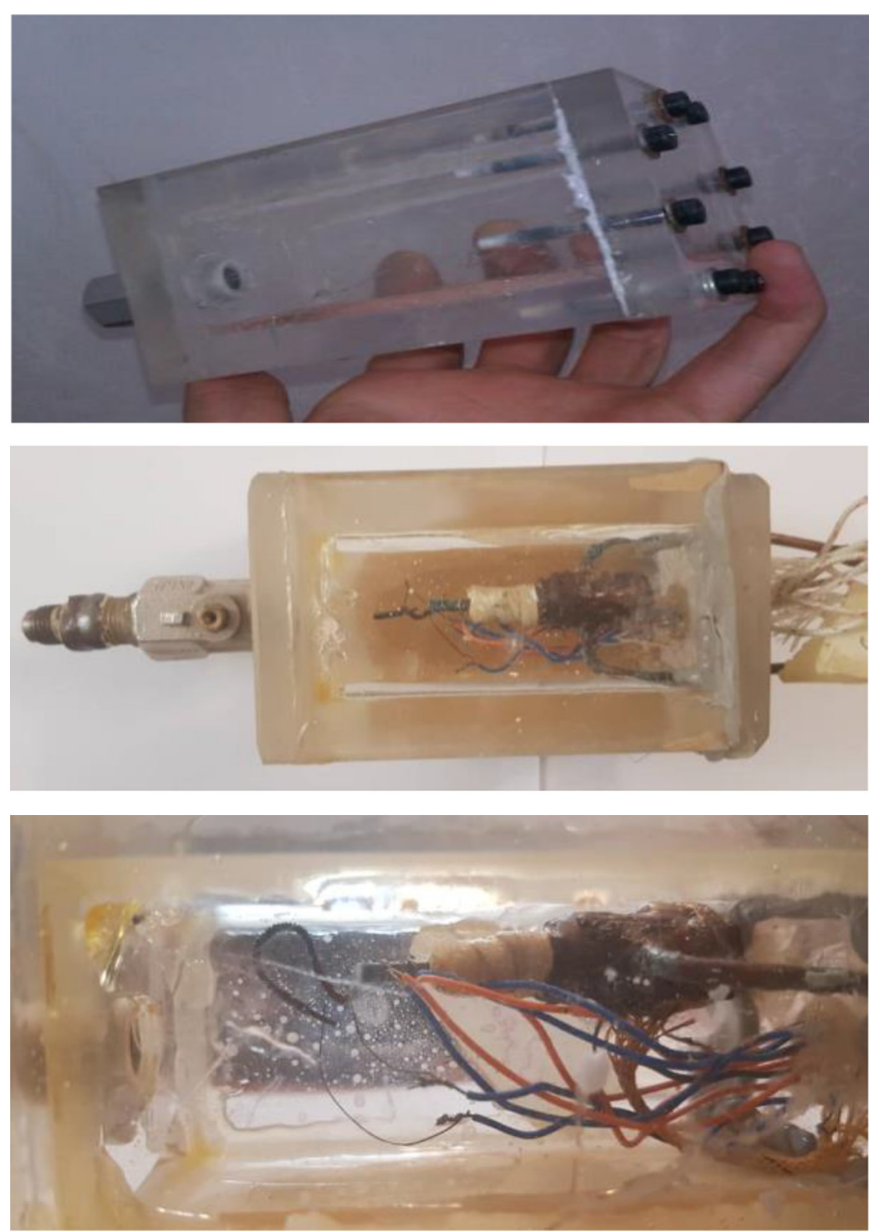

Fig. 5. Location and installation of thermocouple.

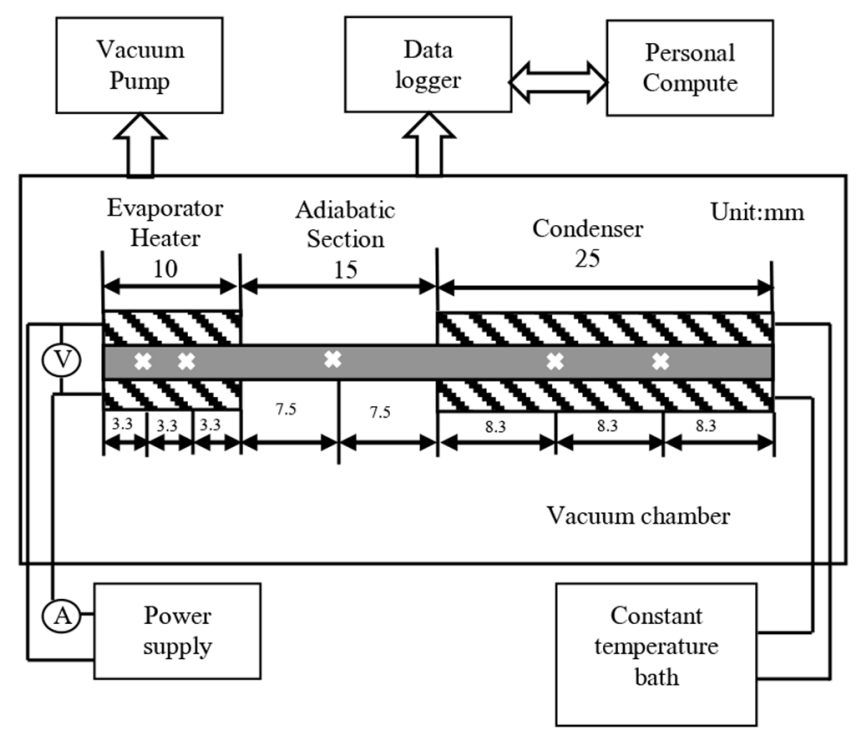

Fig. 6. Schematic of test set up [6].

\section{Working fluid}

In this study, water and different solution mixture of water and ethanol were used as working fluid. Considering that

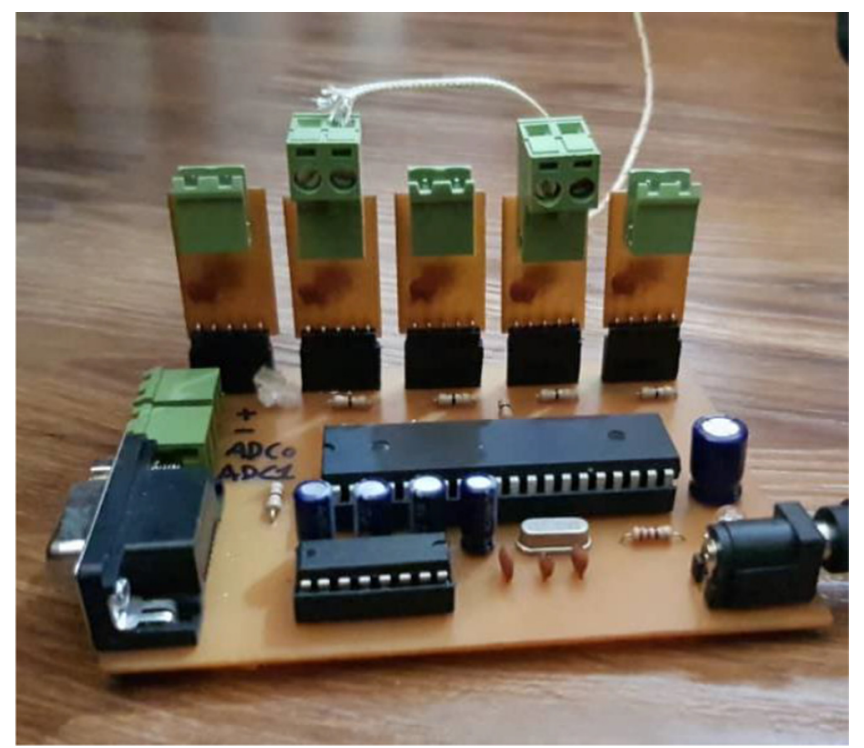

Fig. 7. Micro heat pipe adapter.

the dimensions, very small and the weight of the non-fluids, were considered to be extremely important to obtain a solution with the desired concentration, first a certain volume of water was injected into a laboratory balloon by a pipette. Then the balloon was set to the laboratory scale and set to zero on zero and using the pipette, ethanol was added to the desired concentration. The balloon containing water and ethanol was produced in a magnetic stirring for five minutes (Fig. 8).

\section{Heat transfer characteristics in micro heat pipe}

Heat pipes can transfer a lot of heat via a little temperature difference between the evaporator and condenser. In general, measuring the temperature difference between the evaporator and condenser is one of the test methods to check whether there is a non-compressible gas in heat pipe or a heat pipe works well after the end of the production process. In the micro heat pipe, the production process requires high-precision technologies. Due to the existence of non-condensable gases or pollutants, even trace amounts may be harmful to the performance of heat pipes.

Figure 9 shows temperature distribution in the axial length of $50 \mathrm{~mm}$ in micro heat pipe with triangular and square cross section. Tested MHPs have curved triangular and square cross section with a filling ratio of $20 \%$ of internal total volume. In the condenser, heat was dispersed via conduction heat transfer. Temperature after a steady state is averaged for at least one minute to reduce minor fluctuations error. As shown in Figure 9, MHP wall temperature by increasing the heat load increases. This means that thermal equilibrium, in other word the isotherm properties of MHP from the evaporator to condenser is fulfilled well. The temperature difference between the evaporator and condenser in different thermal loads is shown in the next curves. However, the temperature difference between the evaporator and condenser, 

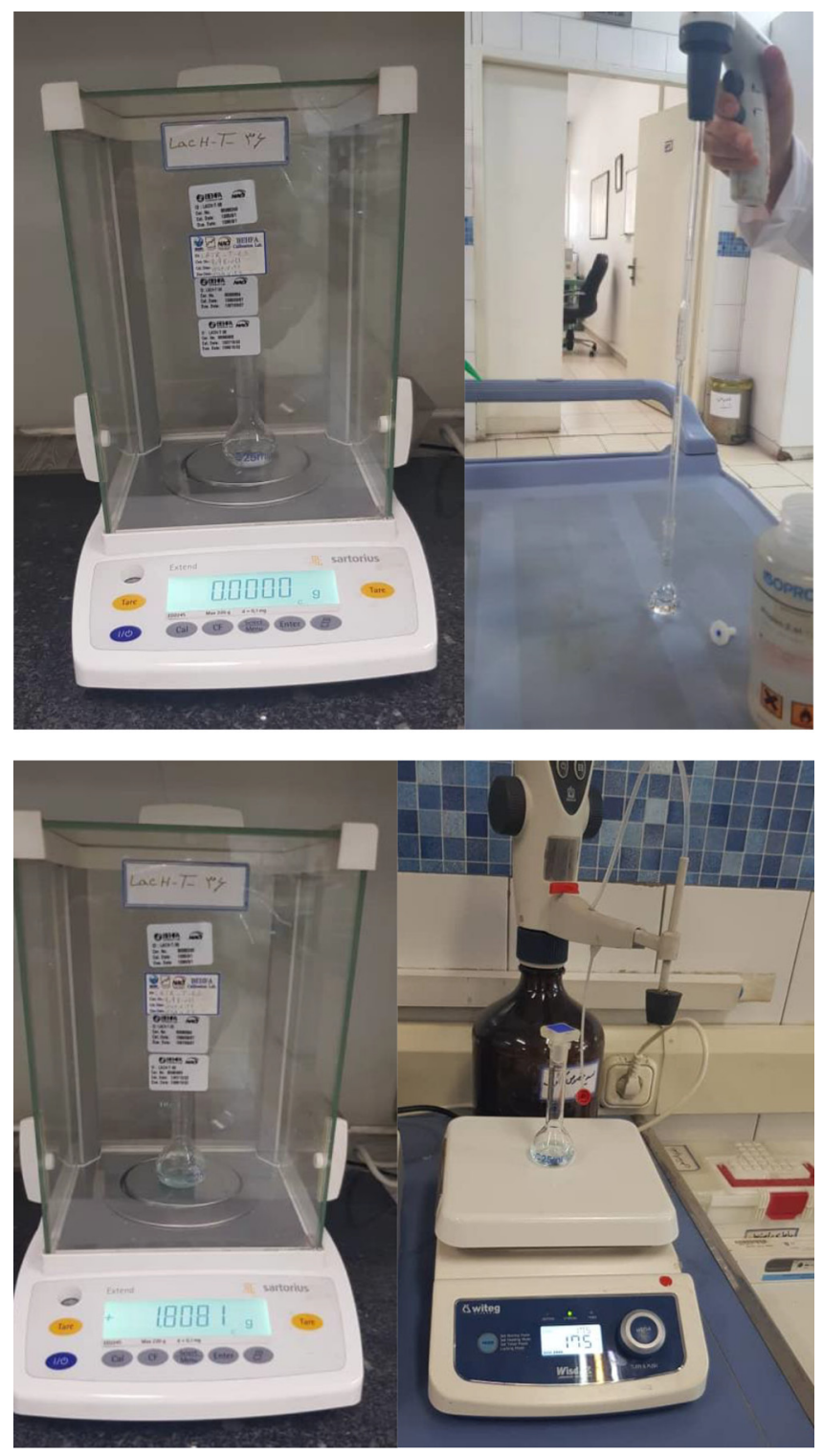

Fig. 8. Steps for preparing the water solution and ethanol.

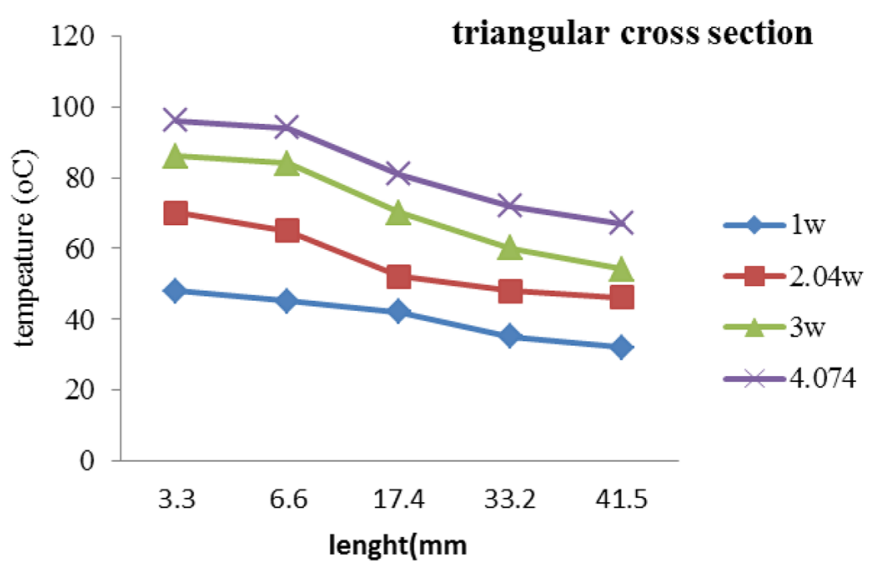

Fig. 9. Temperature distributions in the axial length of $50 \mathrm{~mm}$ in micro pipe with.

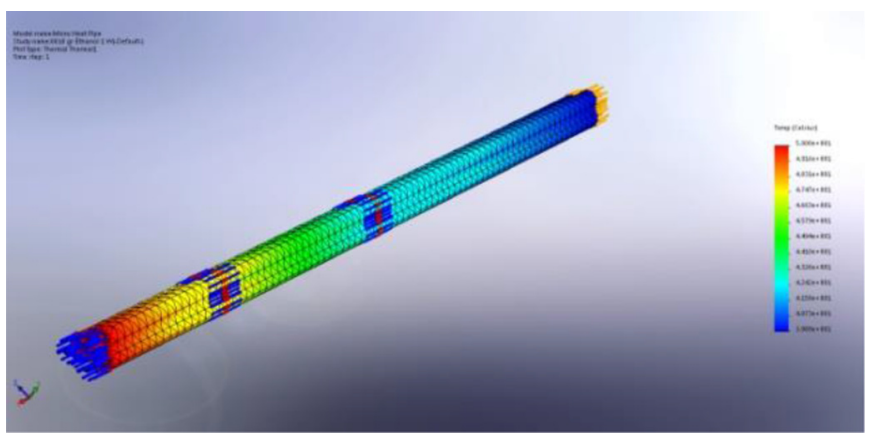

Fig. 10. Meshing that used for boundary conditions.

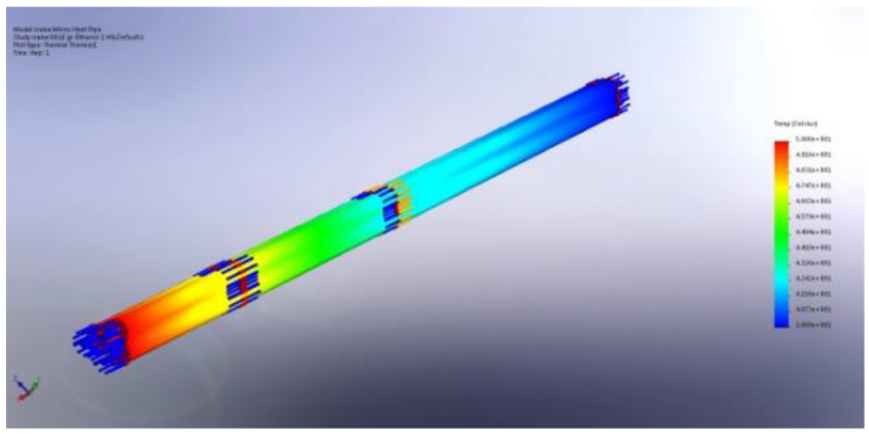

Fig. 11. Thermal contour for input current.

when the working fluid was changed to water and also mixture of water and ethanol was more than that for pure water. This is because the electric double layer leads to disruption of the flow inside the pipe. This due to the small dimensions of the micro heat pipe and also ions of the working fluid influenced performance of micro pipe clearly and result in greater temperature difference between the two section of evaporator and the condenser.

\section{Software modeling}

In addition to experimental tests, micro heat pipe was simulated by the software. It should be noted that the effects of electrical double layer was not considered in this modeling. In simulating the thermodynamic properties of working fluid, the initial conditions and geometry of the micro heat pipeline were considered in accordance with the specimen. The results obtained from the software and the experiments were compared (Figs. 10-12).

\section{Error analysis}

Due to the accuracy of measurement equipment and its effect on the error rate of computational quantities, including Reynolds number, Nusselt number, and heat transfer coefficient, the analysis of these errors is necessary. In this study, Coleman and Steele method is used to calculate the uncertainty of variables. According to this method, if the quantity of $R$ is the function of the variables $x_{1}, x_{2}$ then the uncertainties are derived from the following 


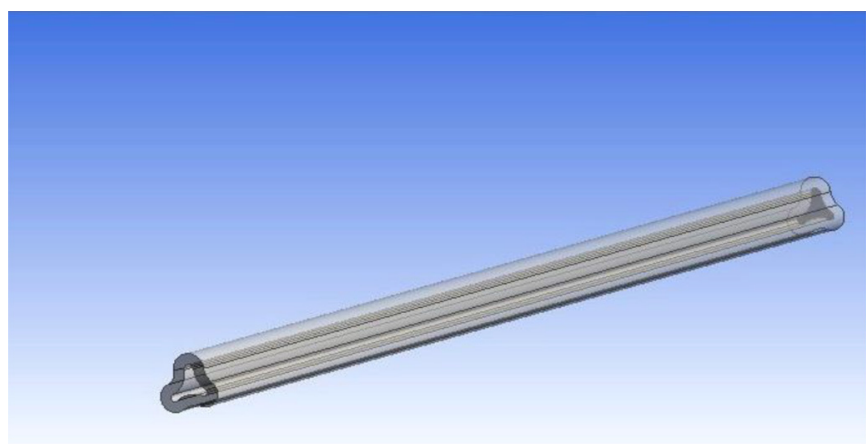

Fig. 12. 3D model of MHP.

Table 2. Accuracy of measurement devices.

\begin{tabular}{lll}
\hline Variable & Measuring tools & Accuracy \\
\hline Pipe diameter & Caliper & $\pm 0.02 \mathrm{~mm}$ \\
Mass & Laboratory digital scale & $\pm 0.1 \mathrm{mg}$ \\
Temperature & Thermocouple Type $\mathrm{k}$ & $\pm 0.5^{\circ} \mathrm{C}$ \\
\hline
\end{tabular}

Table 3. Uncertainty of computational variables.

\begin{tabular}{ll}
\hline Variable & Uncertainty $\%$ \\
\hline Heat transfer coefficient $(\mathrm{h})$ & \pm 9.4 \\
Reynolds number $(\mathrm{Re})$ & \pm 1.7 \\
Nusselt number $(\mathrm{Nu})$ & \pm 11.9 \\
\hline
\end{tabular}

equations:

$$
\begin{aligned}
R & =R\left(x_{1}, x_{2}, \ldots, x_{J}\right) \\
U_{R} & =\sqrt{\sum_{i=1}^{J}\left(\frac{\partial R}{\partial x_{i}} U_{i}\right)^{2}} .
\end{aligned}
$$

The accuracy of the measurement devices in the experimental system is given in the following table presents the uncertainty of the computational quantities (Tabs. 2 and 3$)$.

\section{Discussion and conclusion}

The effect of the cross section shape on the function of micro heat pipes with triangle and square cross-section with thermal loads of 1-4 watts has been studied theoretically and experimentally. Triangular and square micro heat pipes with thin structures were manufactured and tested. Water and different solution mixture of water and ethanol were used as working fluid. MHP which is charged with pure water as working fluid represented

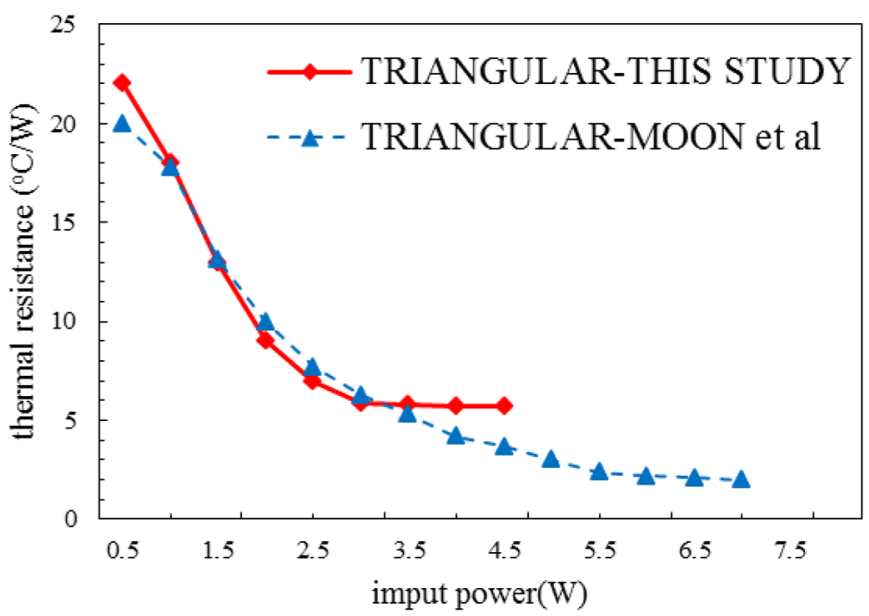

Fig. 13. Validation of sample thermal resistance tested in this case with previous record and researches.

good isotherm properties over pipe length, and the temperature difference between the evaporator and condenser was below $16{ }^{\circ} \mathrm{C}$. Effect of gradient angle on thermal performance was low and thermal characteristics from low heating mode to high heating mode were stable. In use of ordinary water, wall temperature has been higher. This reflects the effect of an electric double layer in the use of ordinary water. MHP which is made in the present study showed a high capacity for heat dissipation and also represented that in used Small scale pipes, the impact of Electrical double layer negatively affected the performance of heat pipes by $20 \%$. Thus it will be able to be used widely in integrated electronic devices as a cooling unit. Triangular Micro heat pipes with the same geometry and working fluid were tested as a reference to investigate the setup and comparing the results with previous reference and studies [15] and the accuracy of regulation of micro heat pipes were confirmed (Fig. 13).

To test the effect of EDL on heat transfer of micro heat pipe, with caring to the influence of alcohol concentration in a solution of water and alcohol, we used various solution alcohol and water in experimental tests and numerical study.

The difference between the experimental and theoretical results in the input power of 1 watt and the solution of water and 0.018 (gr) ethanol was about 0.7 degrees in triangular MHP But this difference in square cross section was far less; of course, the highest dispute in the adiabatic zone was $4 \%$ in triangular cross section (Fig. 14).

As expected, the effect of EDL increased by adding more drops of alcohol to water. By increasing the concentration of ethanol to 3 times in the solution (0.054 gr), the difference between theoretical and experimental results was significantly different in both triangular and square MHPs, so that we observed an average of $5 \%$ of the difference (Fig. 15). At the maximum concentration of the tested solution, 10 drops of ethanol in solution (18 gr), the theoretical results close to the results of low concentrations of ethanol were obtained. While the experimental results 


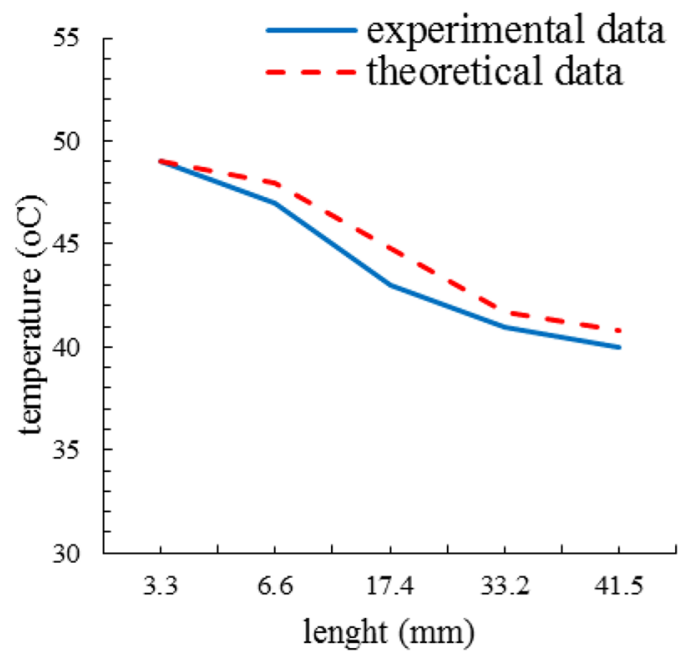

Fig. 14. Compare experimental and theoretical graph of wall temperature, water and $0.018 \mathrm{~g}$ ethanol as working fluid, $1 \mathrm{~W}$.

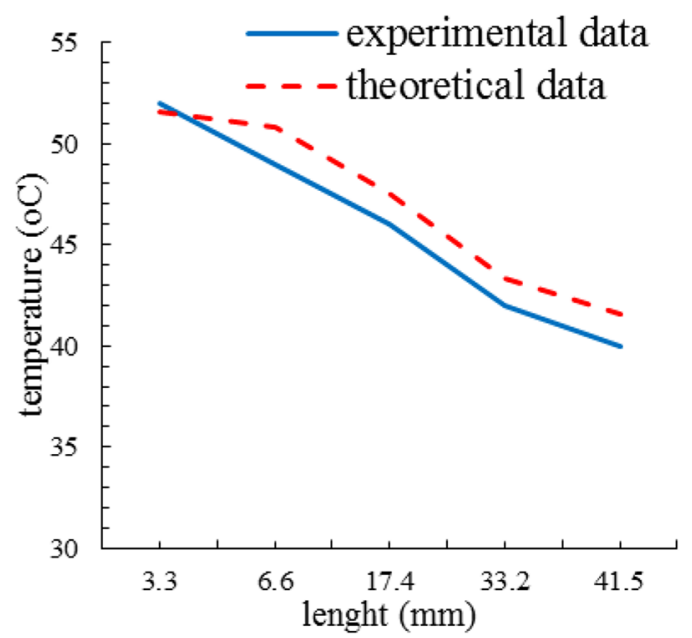

Fig. 15. Compare experimental and theoretical graph of wall temperature, water and $0.054 \mathrm{~g}$ ethanol as working fluid, $1 \mathrm{~W}$.

showed a significant difference between the results at different concentrations, this was due to the EDL effects (Fig. 16).

It was observed the difference between experimental results and extracted data from the theoretical solution have been increased by increasing input power to 4 watt, so in this state the minimum of difference was $2 \%$ and maximum in the worst state was $17 \%$ (Figs. 17-20). But the difference between experimental and theoretical results in MHP with square cross-sectional area due to decreasing effects of EDL because of the geometry of corners was lower than that with triangular cross section.

In both triangular and square MHP, high amount of difference in the EDL influence of 1 or 2 watt input power was not seen. While in the 4 watt input power in addition of differences between experimental and theoretical results because of the existence of EDL factor, temperature

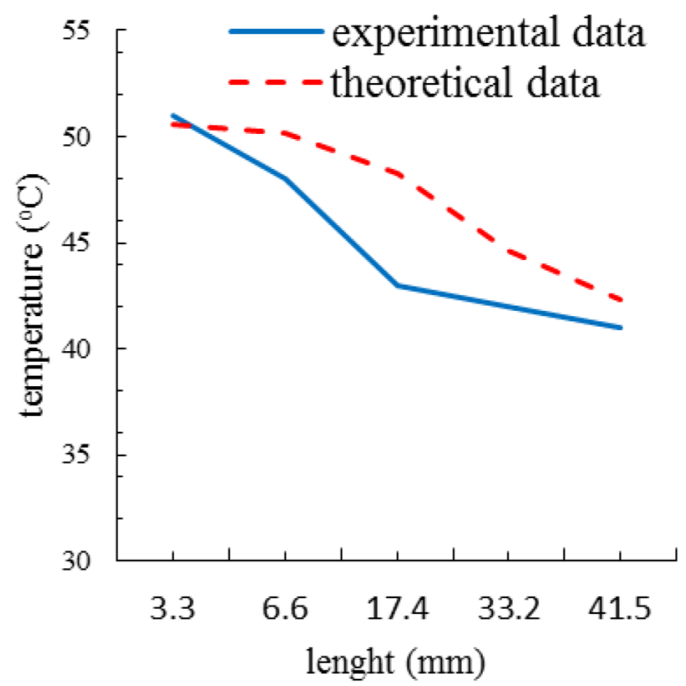

Fig. 16. Compare experimental and theoretical graph of wall temperature, water and $0.18 \mathrm{~g}$ ethanol as working, $1 \mathrm{~W}$.

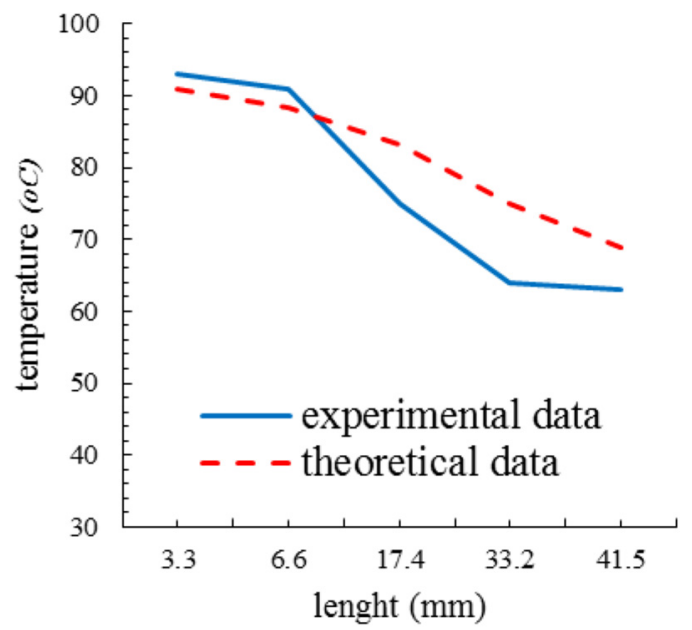

Fig. 17. Compare experimental and theoretical graph of wall temperature, water and $0.018 \mathrm{~g}$ ethanol as working, $4 \mathrm{~W}$.

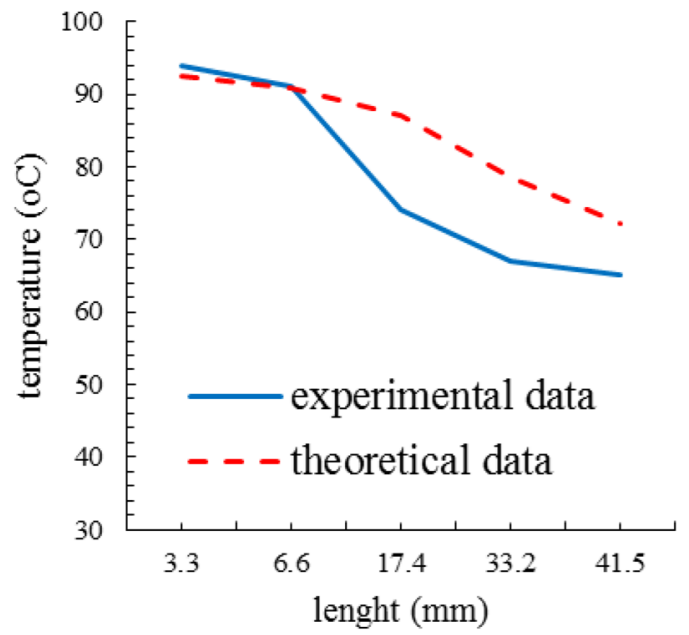

Fig. 18. Compare experimental and theoretical graph of wall temperature, water and $0.054 \mathrm{~g}$ ethanol as working, $4 \mathrm{~W}$. 


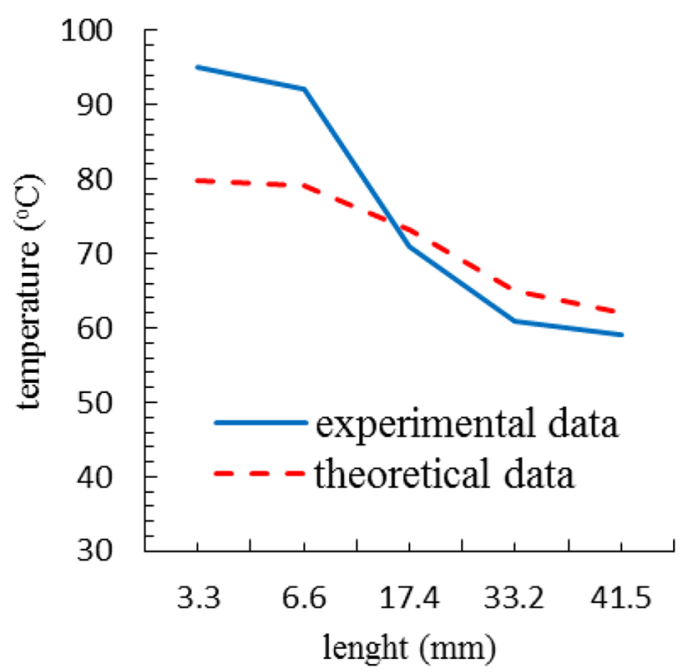

Fig. 19. Compare experimental and theoretical graph of wall temperature, water and $0.18 \mathrm{~g}$ ethanol as working, $1 \mathrm{~W}$.

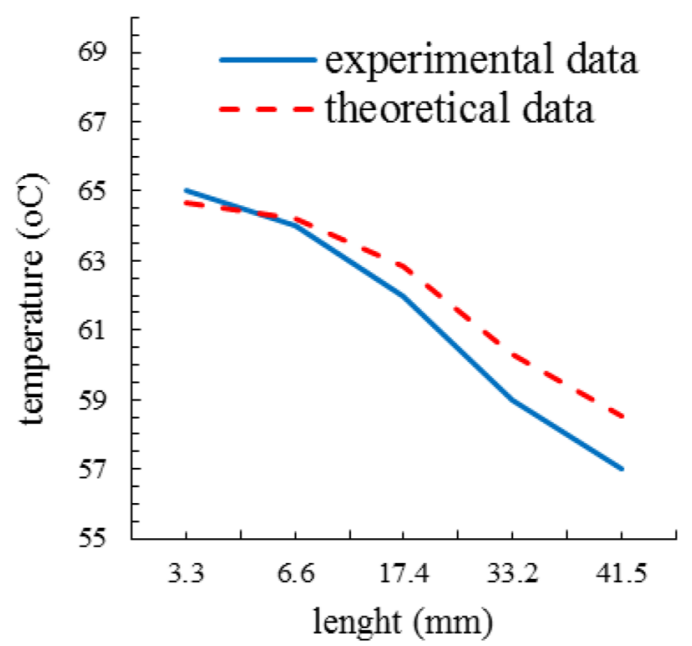

Fig. 20. Compare experimental and theoretical graph of wall temperature, water and $0.054 \mathrm{~g}$ ethanol as working, $2 \mathrm{~W}$.

difference between evaporator and condenser was around $27 \%$; in other words, the existence of EDL has a negative effect on efficiency of both square and triangular cross section MHP. It was noticed that these effects were observed in triangular section due to its geometry more than the square section. Corners in square samples manufactured and tested in this study were normal, but in samples with curved corners such as triangular sample, it is expected that the micro heat pipe efficiency and also the effect of electrical double layer increase. This is due to one additional corner and the increase of the capillary pressure. It is suggested that this issue will be discussed in future researches.

\section{Nomenclature}

$v \quad$ Velocity

$v_{l} \quad$ Velocity of the liquid $v_{g} \quad$ Velocity of the vapor

$v_{r} \quad$ The difference between the velocity of the liquid phase and the vapor phase velocity $v_{l} v_{g}$

$t \quad$ Time

$P \quad$ Pressure

$T$ Matrix transpose

$g \quad$ Acceleration of gravity

$F \quad$ Body force

$f_{1} \quad$ The force caused by an external electric potential

$f_{2} \quad$ Body force generated by an electrical double layer between the liquid and solid

$f_{3} \quad$ Body force generated by an electrical double layer between the liquid and vapor

$E \quad$ Electro viscous force

$k$ The curvature (viewed from the vapor phase)

$\mathbf{k} \quad$ The inverse of Debye length

$k_{b} \quad$ Boltzmann's constant

$\vec{n} \quad$ The unit normal vector of the interface (outward from the gas to liquid phases)

$S_{h} \quad$ Any heating source including radiation and

$k_{\text {eff }}$ Effective thermal conductivity

$T$ Temperature

$q^{\prime \prime} \quad$ At generated by the thermal source

$k_{s} \quad$ Thermal conductivity coefficient of the wall

$C_{p} \quad$ Specific heat

$k$ Inverse of Debye length

F Faraday constant

$R$ Universal gas constant,

$c_{i, 0}$ Bulk concentration of the ith ion

$n_{0} \quad$ Bulk ionic concentration,

$z \quad$ Valence of ions

$e \quad$ Large of a proton

$k_{b} \quad$ Boltzmann's constant

$q \quad$ Charge of the ion

$l \quad$ Fluid

$g$ Gas

$T$ Temperature

Greek Symbol

$\rho \quad$ Density

$\rho_{e} \quad$ Volumetric charging of the double electrical layer

$\mu$ Dynamic viscosity,

$\sigma \quad$ Coefficient of contact surface tension

$\alpha$ Volume fraction

$\phi \quad$ External electrical potential

$\psi_{1}$ Potential of the wall recharge

$\psi_{2} \quad$ Electric potential

$\rho_{e} \quad$ Volumetric charging of the double electrical layer

$\varepsilon \quad$ Dielectric coefficient

$\mu^{S}$ Chemical potential of ions at the contact level

\section{References}

[1] M. Rahmat, P. Hubert, Two-phase simulations of micro heat pipes, Comput. Fluids 39, 451-460 (2010)

[2] G. Mohiuddin Mala, D. Li, J.D. Dale, Heat transfer and fluid flow in microchannels, Int. J. Heat Mass Transfer 40 , 3079-3088 (1997) 
[3] R.W. Knight, D.J. Hall, J.S. Goodling, R.C. Jaeger, Heat sink optimization with application to microchannels, IEEE Trans. Compon. Hybrids Manufact. Technol. 15, 832-842 (1992)

[4] M. Groll, M. Schneider, V. Sartre, M. Chaker Zaghdoudi, M. Lallemand, Thermal control of electronic equipment by heat pipes, Rev. Génér. Therm. 37, 323-352 (1998)

[5] M.L. Berre, G. Pandraud, P. Morfouli, M. Lallemand, The performance of micro heat pipes measured by integrated sensors, J. Micromech. Microeng. 16, 1047-1050 (2006)

[6] M. Fallah Abbasi, H. Shokouhmand, Experimental investigation on effect of EDL on heat transfer of micro heat pipe, Microgr. Sci. Technol. 31, 317-326 (2019)

[7] Y. Lei, Z. Chen, J. Shi, Analysis of condensation heat transfer performance in curved triangle microchannels based on the volume of fluid method, Microgr. Sci. Technol. 29, 433-443 (2017)

[8] K. Fukagata, N. Kasagi, P. Ua-arayaporn, T. Himeno, Numerical simulation of gas-liquid two-phase flow and convective heat transfer in a micro tube, Int. J. Heat Fluid Flow 28, 72-82 (2007)

[9] G. Černe, S. Petelin, I. Tiselj, Coupling of the interface tracking and the two-fluid models for the simulation of incompressible two-phase flow, J. Comput. Phys. 171, $776-804$ (2001)
[10] E. Berberović, N.P. van Hinsberg, S. Jakirlić, I.V. Roisman, C. Tropea, Drop impact onto a liquid layer of finite thickness: dynamics of the cavity evolution, Phys. Rev. E 79, 036306 (2009)

[11] P. Zhang, H.H. Qiu, Investigation of the patterned surface modification on $3 \mathrm{D}$ vortex flow generation in a micropipe, $\mathrm{J}$. Micromech. Microeng. 18, 115030 (2008)

[12] L. Ren, D. Li, W. Qu, Electro-viscous effects on liquid flow in microchannels, J. Colloid Interface Sci. 233, 12-22 (2001)

[13] L. Gong, J.-K. Wu, L. Wang, K. Chao, Periodical streaming potential and electro-viscous effects in microchannel flow, Appl. Math. Mech. 29, 715-724 (2008)

[14] H. Nakajima, Mass Transfer, Open access peer-reviewed Edited Volume (November 4th 2011) (2011)

[15] H. Shokouhmand, A.A.R. Bahrami, Study of the effects of electrokinetic field on heat transfer through rectangular microchannels, J. Fac. Eng. 39, 773-784 (2006)

[16] B.Q.J.A.W. Qian, MEMS-Based Micro-heat Pipes (June 15th 2016)

[17] P. Krata, The impact of sloshing liquids on ship stability for various dimensions of partly filled tanks, TransNav, Int. J. Mar. Navigat. Saf. Sea Transp. 7, 481-489 (2013)

Cite this article as: M. Fallah Abbasi, H. Shokouhmand, M. Khayat, An investigation on effect of EDL on heat transfer of micro heat pipe with square and triangular cross section, Mechanics \& Industry 21, 309 (2020) 